\title{
Émotions et compréhension de narrations littéraires Des rapports si intrinsèquement liés
}

Sophie Roch-Veiras, UCO-LICIA, France

C'était il y a quelques années déjà, alors que je travaillais sur la compréhension de texte avec un groupe d'étudiants étrangers de niveau B1... Mon choix s'était porté sur une narration littéraire. J'attendais que les étudiants aient terminé la lecture du texte... quand l'un d'entre eux a pris la parole et m'a dit : «C'est triste... ».

Je ne sais si sa remarque traduisait une simple affirmation ou s'il souhaitait avoir confirmation... Quoi qu'il en soit, sa réflexion m'a à la fois surprise et intriguée. J'ai supposé qu'il n'attendait qu'un acquiescement de ma part car il semblait avoir compris le texte. Par ailleurs, il était un excellent étudiant. A l'époque, ma brève réponse lui avait permis à l'évidence de confirmer une des hypothèses qu'il avait formulées sur le texte car il s'était tu.

Néanmoins, les émotions et manifestement, celles que les éléments du texte avaient suscitées en lui, semblaient avoir donné valeur de contrôle à sa compréhension du texte. A moins qu'il n'ait tout simplement eu envie de partager avec moi son impression ressentie à la fin du texte. En tout cas, cet épisode m'a fait réfléchir au rôle des émotions dans la lecture et à l'acte de compréhension d'un texte littéraire narratif dans une langue étrangère (LE).

Si les émotions suscitées par les éléments du texte viennent participer à la compréhension, quels liens établir entre les émotions que les éléments du texte suscitent chez le lecteur et sa compréhension en LE? A quel moment de la lecture les émotions interviennent-elles chez l'apprenant? Qu'en est-il du plaisir de lire dans la LE et de l'état émotionnel des apprenants avant la lecture du texte ? Quelles conséquences sur le choix et l'exploitation des narrations littéraires dans un cours de compréhension de texte en LE?

Telles sont les questions qui sont à l'origine de l'étude qui sera présentée ici et auxquelles nous allons nous efforcer de répondre en privilégiant les perspectives issues de regards croisés sur les émotions et la compréhension de texte en psychologie expérimentale et cognitive, ainsi qu'en didactique de la littérature et en sciences du langage.

Comme Martine Abdallah Pretceille l'a affirmé, nous pensons que « la littérature n'est pas réservée aux littéraires et aux enseignants de littérature... Chacun bénéficie d'un droit d'inventaire, notamment les enseignants de langue » (147). Dans cet article où la littérature est au service de la compréhension et des émotions suscitées par la narration littéraire en 
classe de langue, notre intention est de vouloir la «réintégrer dans la perspective d'un apprentissage de l'altérité au service d'un humanisme [...]» (147).

Dans un premier temps, avant de définir le type d'émotions que les éléments d'un texte suscitent, l'aide que ces dernières peuvent apporter à la compréhension, et leur rôle dans la compréhension d'un récit, nous justifierons brièvement notre préférence pour la narration littéraire. Dans un deuxième temps, prenant ensuite comme amorce les émotions du lecteur, nous nous interrogerons d'abord sur le plaisir de lire de l'apprenant, puis sur la lecture littéraire « comme distanciation et comme participation » (Dufays, Gemenne et Ledur 91) et enfin, sur ses liens avec la compréhension d'une narration littéraire. Dans un troisième temps, nous traiterons de la valeur subjective des émotions, communes aux émotions dans la langue et à la narration littéraire, et finalement, au regard de ces recherches, nous proposerons une grille pour une approche par les émotions de la narration littéraire en LE.

Les émotions faisant partie du processus de compréhension, la finalité est qu'à partir d'une approche basée sur les émotions, et en plus de la compétence linguistique, encyclopédique, logique, rhétorique et idéologique définies par Umberto Eco et des compétences stratégiques inhérentes à tout apprentissage, les apprenants puissent développer une compétence émotionnelle.

Nous avons délibérément omis de parler de la compétence de décodage car, à partir du niveau A2/B1, les mécanismes de base doivent en principe être acquis. Néanmoins, nous avons remarqué qu'à ce stade de l'apprentissage, certains étudiants souffraient encore de «déficits » d'automatisation liés à la vitesse de lecture, par exemple. Or, il a été prouvé (Nell ; Just et Carpenter ; Schank) que les émotions exerçaient une influence sur la vitesse de lecture du lecteur.

\section{Émotions et raison : vers un juste équilibre}

Dès l'Antiquité avec Socrate, Platon et Aristote - qui s'était interrogé sur les émotions « pathè » qu'un orateur doit connaitre pour agir sur l'esprit des allocutaires - mais aussi avec les Sophistes, les émotions feront l'objet de réflexions philosophiques. Bell et Darwin marqueront le début d'une approche plus scientifique, mais les émotions, opposées à la raison, seront généralement considérées comme indésirables. Ce ne sera finalement qu'à partir des années soixante avec les psychologues sociaux et cliniciens et surtout, grâce aux récentes découvertes dans le champ des neurosciences, où il a été prouvé que, sans émotions, il ne peut y avoir de rationalité pratique (Damasio), que les émotions cesseront d'être considérées comme un concept marginal. 
Aujourd'hui, nous savons que nous ne pourrions pas concevoir notre vie sans ressentir d'émotions et, dans de nombreux domaines comme ceux de la psychologie, l'émotion, «à mi-chemin entre le corps et la raison, nous a conduits à refermer la parenthèse de l'hommemachine avec la psychologie humaniste»(Blanc 9). En sciences du langage également, l'argumentation dans le discours s'exerce à rechercher désormais dans la trame même des textes l'intrication constitutive du «pathos » et du « logos ».

En didactique de la littérature, les théories de la réception dans les années soixantedix, en réhabilitant l'activité du lecteur, ont permis elles aussi que soit engagée une réflexion sur une approche "lecturale» et sur une lecture "psychoaffective » du texte littéraire (Dufays).

Désormais unies, émotions et raison coexistent, les émotions intéressant de plus en plus de chercheurs dans de nombreuses disciplines. Beaucoup de questions restent cependant posées, notamment celles sur la façon dont les émotions interviennent dans nos apprentissages, et dont elles pourraient être prises en compte, dans l'activité de compréhension d'une narration littéraire.

\section{Texte littéraire, narration et émotions}

Même si dans sa globalité, un texte est caractérisable, il reste difficile à classer car, comme l'écrit Jean-Michel Adam, il est souvent composé de fragments de plusieurs autres prototypes de textes plus ou moins articulés entre eux. La question de la définition d'une typologie des textes pose ainsi problème étant donné la variété générique infinie que peuvent prendre les formes de mise en texte, le texte narratif ne dérogeant pas à la règle. Alors, qu'est-ce qu'un récit? Selon Claude Bremond, proposé par Adam (102), un « récit consiste en un discours intégrant une succession d'évènements d'intérêt humain dans l'unité d'une même action ». «Succession", « intégration dans l'unité d'une même action » et « implication d'intérêt humain » étant des éléments indispensables pour constituer un texte narratif, Bremond précise que c'est seulement «par rapport à un projet humain que les évènements prennent sens et s'organisent en une série temporelle structurée » (102). Par le simple fait que le récit mette en scène un personnage et se rapporte à des évènements vécus par des êtres humains ou anthropomorphes, il génère, par son action et son discours internes au récit, de multiples passions qui permettent au lecteur d'éprouver des sentiments ou des émotions.

Par rapport au texte littéraire, des pistes de définition ont été proposées ; néanmoins, tenter d'en donner une définition claire reste aussi une opération des plus complexes. Pour 
Paule Turmel-John, «tout texte relevant de la fiction, écrit avec un souci d'esthétique et reconnu comme tel par une opinion commune»(51) peut être appelé texte littéraire. Semblable à toute autre narration qui nous permet de vivre dans un monde de fantaisie, de ressentir des expériences humaines et culturelles, que nous n'aurions pas vécues, la narration littéraire se préoccupe de la dimension esthétique d'un texte.

Même si certains étudiants se montrent ou se croient incapables de s'intéresser aux textes à visée esthétique « comme s'ils redoutaient l'illusion littéraire et n'assignaient à ces derniers qu'un rôle purement informatif et pragmatique » (Picard 117), le texte littéraire peut tout à fait trouver sa place dans un cours de LE. Selon Monique de Bonis, « les narrations littéraires constituent ainsi de précieux modèles, dans la mesure où elles nous aident à comprendre la cohérence du monde de nos émotions » (183).

Par conséquent, l'une des actions que peut entreprendre l'enseignant est certainement celle de tenter de faire pressentir à l'apprenant la narration littéraire, « comme source de plaisir accessible » (Gervais 48), un plaisir relié à la richesse des émotions distinctes et variées que les éléments du texte suscitent.

Eu regard à nos objectifs et à notre public, notre étude s'est limitée aux narrations littéraires comportant des récits brefs comme des nouvelles ou contes, et un peu plus longs avec des extraits de roman.

\section{1- A propos des émotions que les éléments du texte narratif suscitent}

\section{1- Les types d'émotions suscitées par un texte}

Les émotions, qui font partie intégrante de l'activité de compréhension et qui apparaissent au fur et à mesure que le lecteur progresse dans le texte, ont un rôle essentiel à jouer dans la compréhension.

On peut en distinguer de deux types, celles liées à des caractéristiques stylistiques, syntaxiques ou sémantiques (par l'intensité affective qui se dégage des termes utilisés) appelées, les émotions " artefacts », qui interviennent plutôt en début et en fin de lecture (Kneepkens et Zwaan) et celles transmises par les personnages présents dans le récit et par les évènements, les émotions «fictives », qui sont davantage ressenties, lorsque le lecteur est plongé dans le récit lui-même, (Meijsing,; Dijkstra et al., cités par Blanc) et qui sont d'autant plus élevées que ce dernier avance dans la lecture du texte. En outre, les émotions peuvent également être véhiculées par le lecteur lui-même qui va alors réagir à sa manière à la situation exposée (Blanc). 
Les émotions « artefacts » qui augmentent selon le niveau de compétences du lecteur et son degré d'expertise en littérature, sembleraient favoriser « une meilleure rétention du texte... parce que le lecteur se rappelle non seulement les émotions ressenties mais également l'objet de ces émotions » (141).

A l'opposé, les émotions « fictives » incitent le lecteur à focaliser son attention sur les informations les plus émouvantes, à traiter les informations de façon plus élaborée et à orienter le contenu de sa représentation. Par rapport à ce type d'émotions, et concernant l'«empathie » du lecteur pour le protagoniste, le lecteur n'a pas besoin de s'identifier au protagoniste pour représenter ses états émotionnels; il peut être simplement un témoin privilégié et «réagir émotionnellement à ce qu'il sait de la situation exposée » (Tan et Schneider cités par Blanc 142).

Selon Gernsbacher et al., c'est dès le début de la lecture que le lecteur, et ce de façon automatique, s'engage dans la représentation des états émotionnels du protagoniste du récit en se basant sur ses connaissances émotionnelles qui peuvent être propres à lui-même ou bien issues de sa mémoire sémantique. La représentation des états émotionnels du protagoniste du récit s'élabore ensuite continuellement à partir de la construction d'hypothèses sans cesse vérifiées ou infirmées. A chaque fois que le lecteur a représenté l'état émotionnel du protagoniste, toute nouvelle information est évaluée compte tenu de la présence de cette information émotionnelle. Selon que les évènements décrits sont en adéquation avec cet état émotionnel, « la représentation devient plus accessible, si elle l'est moins, une nouvelle émotion est activée » (Blanc 171).

Comme l'attestent les recherches de Nathalie Blanc et ainsi que nous avons pu le vérifier chez les apprenants qui lisent un texte en LE, les émotions interviennent aussi sur la vitesse de lecture de ces derniers qui ralentissent au moment où ils sont en train de lire un passage qu'ils estimeront être leur préféré. La cadence de lecture, à l'inverse, peut s'accélérer si ces mêmes lecteurs, jugeant le passage excitant, sont pressés de connaitre la suite.

De ces travaux, il ressort que les émotions ont l'avantage considérable de pouvoir aider le lecteur «à activer l'information pertinente et à décider de l'importance de la situation » (184). Chez un lecteur moyen pour qui il n'est pas encore possible de sélectionner de façon efficace et sans peine l'information importante, il est d'ailleurs probable que les émotions puissent jouer un rôle de remplacement. L'« impression émotionnelle », telle qu'elle est nommée, permet alors au lecteur d'exercer un contrôle sur son processus de lecture, surtout au début d'un texte, quand il y a peu d'indices liés au texte. (Miall ; Spiro ; van Dijk et Kintsch). 


\section{2- Leur aide pour la compréhension de texte}

Issu d'un questionnaire que nous avons effectué en 2008, auprès d'une quarantaine d'étudiants étrangers (de niveau A2 à C1) et de Français qui apprennent une LE, il ressort que les émotions que les éléments du texte suscitent, rendent la recherche d'indices plus facile pour l'apprenant, qui peut ainsi se focaliser sur les idées principales. Elles lui donnent également la possibilité de construire du sens et, faisant en sorte que sa réflexion sur le texte soit activée, facilitent chez lui l'élaboration d'hypothèses. Elles lui permettent aussi de développer plus d'empathie par rapport au texte. De plus, en lui faisant révéler chez lui des évènements inattendus et surprenants, l'imagination de l'étudiant, grâce aux émotions, apparait davantage activée ; son anticipation imaginative l'est également car les émotions éveillent aussi sa curiosité de lecteur par rapport au texte. En outre, elles l'incitent à établir des liens avec ses connaissances et ses expériences émotionnelles en suscitant chez lui, des prises de conscience. Les émotions aidant l'apprenant à prendre de la distance par rapport au texte, elles exercent aussi sur lui un contrôle sur son processus de lecture. Finalement, elles lui permettent de maintenir son attention et d'évaluer sa difficulté par rapport au texte car si l'étudiant ne comprend pas ce dernier, il ne pourra pas ressentir d'émotions.

\section{3- Qu'en est-il de leur rôle dans le cas de la compréhension d'un texte narratif?}

Dans la compréhension d'un récit, les deux types d'émotions sont interdépendants. Un lecteur peut, par exemple, ressentir une émotion liée aux évènements ou aux personnages, c'est-à-dire une « émotion fictive », qui l'amènera peut-être à éprouver de l'admiration pour l'auteur, c'est-à-dire une " émotion artefact ».

Rejoignant l'hypothèse de Nathalie Blanc, nous avons aussi constaté que, quel que soit le niveau de compétences de l'apprenant dans la langue, les « émotions artefacts » étaient plus difficiles à comprendre et à représenter. Des étudiants chinois interrogés ont dit qu'en français les « choses » étaient dites indirectement, par les actions, les temps, la grammaire.

$\mathrm{Au}$ cours de la lecture, en fonction de l'évolution du ressenti sur le texte, les apprenants adoptent des postures de lecteurs différentes: certains s'identifient au protagoniste alors que d'autres restent des témoins privilégiés de la situation. Pour d'autres encore, c'est du lien entre le texte narratif et leurs expériences propres, ou de leur vécu culturel que se dégagent des émotions.

Entre émotion et approche cognitive, la lecture littéraire qui place le lecteur au cœur de ses préoccupations théoriques et didactiques en réhabilitant ce dernier, met en jeu un sujetlecteur, l'apprenant, dans ses rapports psychoaffectifs avec la lecture et la littérature. Situées à la charnière de la compréhension de texte, de la narration, de la lecture d'œuvres littéraires 
et des émotions - nos recherches, qui appellent un traitement pluridisciplinaire et dont les enjeux ont pour finalité la classe de langue, nécessitent qu'un éclairage soit fait sur certaines connaissances afin que des liens puissent être établis.

\section{2- Narration littéraire et émotions du lecteur}

\section{1- Brefs rappels théoriques en matière de lecture littéraire et de compréhension des narrations}

Se référant à une approche phénoménologique, sémiotique, pragmatique voire psychanalytique de la lecture des œuvres littéraires et rompant avec « une littérature axée sur la seule littérature » (Dufays 79), la lecture littéraire « est aujourd'hui utilisée pour désigner une lecture riche, intense, plurielle, à la fois ludique et lucide, passionnelle et rationnelle, qui ne se réduit pas à la littérature »(Dufays, Gemenne et Dedur 11). Elle renvoie à un ensemble de pratiques qui intègre le lecteur dans sa subjectivité et sa singularité : "Chaque œuvre littéraire engendre ainsi une multitude d'œuvres originales produites par les expériences à chaque fois uniques des lecteurs empiriques » (Langlade 87). La lecture littéraire s'appuie également sur des conceptions différentes mais aussi complémentaires de la lecture, l'une privilégiant la lecture comme «mise à distance», l'autre, comme «participation psychoaffective » et la dernière, comme «va-et-vient dialectique », à mi-chemin entre les deux.

En matière de compréhension de textes écrits, les travaux effectués en psychologie cognitive depuis une trentaine d'années ont mis, d'une part, en évidence le fait que la compréhension est un processus de construction dynamique qui aboutit à l'élaboration d'un « modèle mental de situation dans lequel l'information du texte est élaborée, interprétée à partir des connaissances préalables des lecteurs, et intégrée dans celles-ci» (Golder et Gaonac'h 115). Concernant les narrations, ce schéma mental, constitué de façon progressive par le lecteur, contient les personnages de l'histoire, leur statut, leurs relations, leurs buts poursuivis, l'interprétation de leurs actions, les résultats qui en découlent et pourquoi. Comme l'indiquent C. Golder et D.Gaonac'h, « la représentation que le lecteur construit du texte résulte de fait, de la reconstruction de ce dernier » (118).

\section{2- A la croisée des chemins...}

Dans la perspective de la psychologie cognitive, « comprendre un texte, c'est décoder les mots, et c'est aussi en parallèle mettre en œuvre un ensemble de traitements cognitifs de haut niveau pour rétablir les informations implicites, et ainsi construire une représentation cohérente, en convoquant les connaissances préalables du lecteur » (117). Allant plus loin, 
Caroline Golder et Daniel Gaonac'h ajoutent que «lire, c'est interpréter » (118). Alors, si trois lecteurs différents lisent le même texte, «il en résultera forcément trois représentations différentes, les écarts entre les représentations pouvant être attribués à la connaissance antérieure des lecteurs, à leurs objectifs de lecture, à leurs intérêts, etc. » (118).

De même, selon le point de vue de la lecture littéraire, la lecture est décrite comme « un jeu » qui, comme tout jeu, est soumis à des règles (Picard) et où l'auteur du texte «présuppose la compétence de son Lecteur-Modèle, en même temps qu'il l'institue » (Eco cité par Rouxel). Selon Annie Rouxel, le sens est «pluriel dans la synchronie comme dans la diachronie, et se construit dans l'interaction texte et lecteur»(9). Enfin, pour Catherine Tauveron, «Lire de la littérature, c'est produire du sens (et non seulement le recueillir) en collaboration avec le texte, c'est-à-dire dialoguer avec lui en tenant compte de ce qu'il est » (26).

Parallèlement à ces recherches, d'autres, également en psychologie cognitive, stipulent que, simultanément à l'acquisition des opérations constitutives de la compréhension, pour devenir un «compreneur » efficace, le lecteur doit mettre en œuvre des connaissances relatives à des stratégies de compréhension. Ces stratégies, qui favorisent une lecture active et autorégulée et dont le savoir-faire nécessite d'adopter une démarche métacognitive, place l'apprenant dans une attitude d'implication et de distanciation, posture indispensable pour comprendre un texte.

De la même façon, dans le type de jeu réclamé par Michel Picard, la lecture implique identification et distanciation. Picard distingue d'ailleurs trois instances lectrices chez un même lecteur, identités dont il dit qu'elles se superposent et interagissent. Ainsi,

le liseur est la personne physique qui maintient sourdement le contact avec le monde extérieur, le lectant désigne l'instance intellectuelle capable de prendre du recul pour interpréter le texte et le lu renvoie à l'inconscient du lecteur qui réagit au texte et s'abandonne aux émotions si bien que l'on peut dire que la personnalité du lecteur est "lue", révélée, par le texte. Dans l'activité de lecture, ces trois instances interfèrent en un jeu subtil de participation et de distanciation, le liseur et le lu fondant la participation et l'investissement fantasmatique du sujet lecteur et le lectant instaurant une distance avec le texte. (cité par Rouxel 15)

En résumé, aussi bien les recherches sur la lecture littéraire que celles sur la compréhension de textes en psychologie cognitive supposent le fait que tout lecteur se réfère à un modèle qui lui est propre et qu'il reconstruit. Comme pour un « jeu » avec des règles, cette nécessaire reconstruction impose à l'apprenant qu'il soit actif : un apprenant, qui, lorsqu'il se lance dans la lecture du texte, aborde cette dernière avec ses structures cognitives 
et affectives, et qui, pour comprendre, se voit contraint, tout à la fois, de s'impliquer dans le texte et de se mettre à distance par rapport à ce même texte.

\section{3- " Plaisir " et " déplaisir " de lire en LE}

Pour de nombreux adultes qui s'adonnent à la lecture, celle de récits ou de romans littéraires est essentiellement liée à la notion de «plaisir ». A l'opposé de l'« ennui », de la « colère » ou de la « peur » par exemple, le «plaisir » est une émotion positive. Le « désir » l'est également. Néanmoins, en tant qu'émotion d'anticipation, le « désir » consiste à nous faire imaginer une situation concrète qui nous procurerait $\mathrm{du}$ " plaisir » et nous permettrait d' « éprouver de l'excitation dans l'attente de sa réalisation » (Larivey 65), contrairement au « plaisir » qui n’est satisfait que lorsqu'il est comblé.

Alors, qu'est-ce qui procure le « plaisir » de lire ?

Dans sa conception de la lecture psychoaffective, Poslaniec (cité par Dufays) estime que le plaisir de lire, plaisir immédiat, est provoqué par la capacité qu'ont les textes à nous surprendre et à captiver notre imagination. Monique Lebrun, Victor Guérette et Pierre Achim affirment que ce plaisir est lié à «l'expérience esthétique » qui vise à l'imagination et qui contribue à l'émancipation du lecteur. Annie Rouxel définit ce «plaisir esthétique » comme « un plaisir complexe, propre de l'activité de lecteur et du plaisir du texte. Ce dernier est à la fois plaisir du texte et plaisir de la reconnaissance : il nait de la tension entre le dépaysement lié à l'inconnu du texte et le sentiment de familiarité que confère la reconnaissance de codes, le partage de références » (19).

Selon Picard, le plaisir du lecteur nait de l'alternance entre la participation de ce dernier et sa distanciation; en revanche, il estime que c'est la posture distanciée qui suscite le «plaisir esthétique ». W. Iser (1985) a montré que le plaisir de la lecture était lié à la créativité. Dans ses travaux, W. Kintsch (1980) a traité de la notion de "plaisir cognitif», apparentée à la curiosité, qui nait de la réalisation d'un schéma activé (et confirmé ou non) par l'apprenant, à un moment donné au cours de la lecture.

Dans une classe de langue, plus qu'à l'ordinaire, la notion de plaisir revêt un caractère complexe. Chez l'apprenant, en général, cette notion est soumise à de nombreuses contraintes parmi lesquelles la préférence pour un genre de textes (narratif, informatif, prescriptif...), la position de lecture adoptée par le lecteur (utilitaire ou esthétique) ou le type de projet (lecture pour soi ou extérieur à soi), mais aussi les circonstances matérielles (comme le moment ou le lieu de la lecture) etc.

Pour un apprenant de LE, le plaisir éprouvé par la lecture d'un texte dans sa langue maternelle est souvent différent de celui ressenti dans la LE car «l'inconnu» et «le 
dépaysement », auxquels Rouxel fait allusion, et qui sont habituellement liés à la notion de «plaisir, sont souvent à l'origine d'une « peur » chez lui. Dans de nombreuses situations de lecture, l'« échec interprétatif programmé », à l'origine du plaisir, selon Umberto Eco, ne sera donc pas vécu comme une source de plaisir mais il sera plutôt ressenti comme du « déplaisir », ce quel que soit le niveau, comme nous avons pu le remarquer chez la majorité des étudiants de FLE (interrogés lors d'une enquête en 2011).

Cette peur de ne pas comprendre chez l'étudiant, liée à des compétences amoindries ou à un manque de confiance en ses capacités d'apprentissage, réside d'ailleurs souvent dans un « état de crainte anticipatoire », déclenché par l'imagination (Bandura 356). Ainsi, lorsque l'apprenant de LE fait des prédictions sur ce qu'il va lire et qu'il active ses connaissances sur le type de texte, le "plaisir» de lire dans la LE passe de fait souvent au second plan. Si, néanmoins, au cours de la lecture, l'apprenant a maintenant une bonne compréhension du texte, l'«ennui » fera place à la «curiosité »: «piqué au jeu, il se conduit alors en découvreur du texte et, concerné, s’y construit »(Picard 48).

En amont de la lecture, le plaisir de lire devrait être indispensable, mais même s'il l'est, nous savons aussi comme l'attestent Jean-Louis Dufays, Louis Gemenne et Dominique Ledur que le seul plaisir de lire ne présage pas d'une bonne compréhension du texte. Ainsi, « il est bon aussi que ce plaisir ne survienne qu'après coup, au cours de l'exploitation collective du texte en classe, dont il est alors le résultat et le signe de son efficacité »(145). Dans ce cas-là, une des fonctions de l'enseignant est probablement celle d'être un « éveilleur de plaisir » (Serres cité par Dufays, Gemenne et Ledur 145).

A ce propos, nous ajouterons que les émotions ressenties pendant la lecture du texte dépendent aussi de l'état émotionnel initial du lecteur. Blanc et Tapiero (2000) ont même démontré que l'état émotionnel dans lequel se trouve le lecteur avant même la lecture l'oriente dans sa sélection des informations.

\section{3- Les émotions : une porte d'entrée pour la compréhension de la narration littéraire dans la classe de langue}

Plus haut, nous avons d'abord voulu montrer que les émotions du lecteur s'inscrivaient dans un processus de reconstruction de sens. Ainsi, dans un récit, par exemple, le lecteur, pour comprendre et anticiper les comportements et réactions de chacun, se voit obligé de suivre chaque émotion. Nous retiendrons également le fait que le rapport que le lecteur établit avec la narration littéraire réclame à la fois un investissement psychoaffectif et une posture distanciée, et que lire un texte exige une participation dynamique du lecteur qui 
«ne subit pas sa lecture mais la produit» (52) d'autant plus lorsqu'il s'agit d'un texte littéraire.

La plupart des étudiants qui intègrent les cours ont une expérience, parfois lointaine, de la lecture de narrations littéraires dans leur LM et dans la LE. Selon les études qu'ils ont effectuées, la maitrise des techniques littéraires, plus ou moins consciente, varie chez chacun d'eux.

\section{1- De la valeur subjective des émotions}

En linguistique, une classification au niveau lexical, morphologique et sur le plan de la construction syntaxique a été effectuée par Kerbrat-Orecchioni (2000). Par ailleurs, de nombreuses recherches en analyse du discours, sur les émotions dans la langue, comme celles par exemple sur les adjectifs psychologiques (Anscombre) ou celles sur les verbes psychologiques (Plantin) ont été réalisées. Même si ces divers travaux peuvent avoir un impact sur la réflexion par rapport aux émotions et à l'activité de compréhension d'un texte dans la classe de langue, organiser les émotions en un "système linguistique des émotions " (107) est infaisable, étant donné, comme le font remarquer Catherine Kerbrat-Orecchioni et Christian Plantin, leur subjectivité langagière.

La littérature étant d'abord « une manière de lire », Jean-Louis Dufays (84) a souligné la «valeur relative» du texte littéraire tandis que Ruth Amossy a mis en évidence la complexité de la construction des émotions dans le discours. En effet, pour susciter et construire discursivement des émotions, l'émotion peut être soit «mentionnée explicitement » soit « provoquée sans être désignée » (167).

Si elle n'est pas explicitée, il est clair que l'émotion suscitée par le texte sera certainement plus difficilement accessible pour un apprenant de LE. Plantin, pour essayer de voir ce qui peut provoquer une réaction affective a suggéré une situation identique, sans sentiments exprimés. Il a ainsi remarqué que, quand il n'est fait aucune référence à un champ lexical relatif aux émotions, ce sont « des normes, des valeurs, des croyances implicites qui sous-tendent les raisons, qui suscitent des émotions » (Amossy 168). Le texte contient, à ce moment-là, une «topique », associée à des lieux, par exemple, qui dans notre culture justifient une émotion.

Quand les émotions sont exprimées, indépendamment des champs lexicaux, les émotions peuvent aussi se dire dans des procédés syntaxiques (ordre des mots, phrases exclamatives etc.) ou dans les marques stylistiques (le rythme, l'emphase, les répétitions etc.). 
Plantin a également rendu compte d'autres difficultés liées notamment à la « source d'émotion » et au « lieu psychologique » ou « siège de l'émotion ».

L'énoncé d'émotion attribue une émotion à une personne et, dans certains cas, mentionne la source de l'émotion. Ce modèle est linguistiquement fondamental, dans la mesure où la relation d'émotion (source-lieu-émotion) correspond à la structure sémantique d'une famille d'énoncés élémentaires. Il assigne à l'analyse de la parole émue la tâche fondamentale de déterminer qui éprouve quoi - et éventuellement pourquoi (108)

Dans l'exemple où une personne rapporte un évènement à une autre qui n'y a pas assisté, le discours de la personne à qui on raconte l'évènement «induit » des émotions sur celle qui le raconte et qui, en « rejouant» ou en « revivant» (104) les émotions primitives, éprouve des émotions nouvelles. On peut imaginer «la complexité de l'articulation des sources d'émotion dans le récit d'émotion, et la rétroaction systématique de la communication sur le communiquant source de l'émotion » (104).

La définition du «qui » ou du siège de l'émotion peut aussi être à l'origine de confusion, comme dans la phrase suivante, par exemple, où aucun siège psychologique n'est exprimé : «Dans l'énoncé, Luc est un répugnant personnage, le sentiment de répugnance ayant pour source Luc a pour siège le locuteur, et par défaut ou par empathie, l'interlocuteur (c'est-à-dire l'interlocuteur idéal) » (109)

Ces quelques exemples parmi tant d'autres que nous ne pourrons pas tous évoquer, nous montrent que les récits d'émotion et l'assignation de l'émotion peuvent être la cause d'ambigüité et de subjectivité.

D'ailleurs, les émotions, « qui reposent sur des croyances et des jugements, sont ellesmêmes ouvertes à l'argumentation» et «peuvent être argumentées par des locuteurs qui cherchent tantôt à les justifier, tantôt à les délégitimer » (Amossy 165).

Partant de ce principe-là, la discussion, «sur» et «à propos » des émotions dans la narration littéraire, au sein de la classe, ne peut être envisagée que, comme un acte de partage des émotions entre les participants où les émotions ressenties seraient «argumentables » et comme telles, se devraient d'être argumentées (voir infra).

\section{2- Propositions générales}

Selon Mendlevitch (cité par Blanc) et ainsi que nous avons pu le constater, la construction d'une représentation est plus riche pour les textes littéraires où l'émotion reste implicite que pour ceux où l'émotion est explicitement mentionnée.

Pour commencer, l'enseignant doit d'abord « inviter les apprenants à se préparer à une expérience subjective qui explore des possibilités variés plutôt qu'à une expérience objective 
centrée sur la recherche de la bonne réponse » (Giasson 112). Au début de l'apprentissage, il convient de proposer des textes linguistiquement faciles pour que les apprenants puissent apprendre à construire la cohérence des situations proposées, en s'aidant des émotions, comme indices.

En amont, les émotions interviennent dès le moment où l'enseignant choisit les contenus à enseigner. L'activité proposée pourrait ainsi se concevoir de la façon suivante : l'enseignant distribue d'abord à tous les apprenants, trois voire quatre textes, selon la longueur. Chacun les lit et en choisit un. Par groupe de trois, les étudiants travaillent ensuite sur le texte choisi, à partir de la grille proposée ci-après. Enfin, chaque groupe exprime les émotions qu'il a ressenties par rapport au texte, les expose, les partage et explicite ses arguments aux autres étudiants, qui auront d'ailleurs peut-être opté pour le même texte, et qui ne doivent pas hésiter à intervenir et à proposer leurs arguments.

Comme le souligne Tauveron, c'est « dans la confrontation aux autres que se pense, s'affine, se corrige ou se réoriente la lecture de chacun, que se libèrent les potentialités du texte » (26), le partage social des émotions « favorisant la cohésion du groupe » (Espitalier, Tcherkassof et Delmas 94) y compris, celle du groupe-classe.

\section{3- Une approche par les émotions de la compréhension des narrations littéraires}

Le document que nous proposons, intitulé «Une approche par les émotions de la compréhension des narrations littéraires », est une grille que nous avons élaborée et expérimentée avec des étudiants de FLE (B2/C1). La grille n'est pas distribuée telle quelle. Auparavant, des explications auront été données. Par ailleurs, les apprenants auront aussi discuté, à partir d'un questionnaire qu'ils auront rempli, de leurs habitudes de lecture et de leurs comportements de lecteur, dans leur LM et en français, cette activité n'excluant pas d'autres d'ailleurs, sur d'autres genres de textes, et par rapport à l'acquisition de stratégies variées pour la compréhension en LE. Tout comme Dufays, nous pensons que « la classe devrait devenir un lieu où plusieurs lectures littéraires d'un même texte, celles des différents élèves et d'autres dont le professeur fournit des modèles, participent à l'élaboration du sens » (144), cette grille de lecture ne constitue donc qu'un échantillon parmi d'autres. 


\section{« Grille » de la compréhension des narrations littéraires par les émotions}

\section{Votre état émotionnel}

Comment vous sentez-vous aujourd'hui ? Au moment où vous avez lu le texte quel a été votre état émotionnel avant de commencer la lecture ? Positif ou négatif ? Avez-vous ressenti des émotions ? Lesquelles? Et maintenant? Votre anticipation par rapport à la situation de lecture de ce texte a-t-elle induit chez vous des émotions positives ou négatives? Lesquelles? Pourquoi?

\section{Votre impression émotionnelle par rapport au début du texte}

Après avoir lu le titre (et/ou le début du texte), quelles impressions ressentez-vous ? Quels éléments du texte vous donnent cette impression ? Les lieux, les moments, la description des personnages, le style du texte, la syntaxe, le vocabulaire... Notez tous les indices.

\section{A propos de vos émotions suscitées par les personnages principaux}

Ressentez-vous des émotions par rapport aux personnages principaux ? Lesquelles ? Ce sont les informations écrites dans le texte qui vous ont permis de comprendre les émotions que vous avez ressenties par rapport aux personnages du texte ? Ce sont des indices que vous avez trouvés dans le texte, qui ont fait que vous ayez vous-même construit vos émotions sur les personnages ? Ce sont des réponses que vous avez su construire dans votre tête, grâce à des connaissances que vous aviez déjà sur l'histoire (ou le thème de l'histoire) ou à des connaissances liées à vos expériences personnelles ou culturelles, qui vous ont fait ressentir ces émotions-là par rapport aux personnages ? Relevez tous les éléments du texte qui ont suscité chez vous ces émotions. Vos émotions par rapport aux personnages ont-elles évolué ? Pourquoi ? Relevez tous les éléments du texte, relatifs aux personnages qui montrent que ces émotions ont évolué. Vous êtes-vous identifié au protagoniste ou êtes-vous resté témoin de la situation?

\section{Vos émotions suscitées par les lieux}

Ressentez-vous des émotions par rapport aux lieux nommés ou évoqués dans le texte ? Citez les endroits, nommez les lieux (explicites ou implicites) et expliquez pourquoi vous ressentez ce type d'émotions. Dites si vous connaissez ces endroits ou ces lieux, et si vous les avez déjà rencontrés? Dites si ces lieux vous en rappellent d'autres... que vous avez aimés ou détestés. Quelles émotions évoquent-ils pour vous?

\section{Vos émotions suscitées par les moments}

Ressentez-vous des émotions par rapport aux moments nommés ou évoqués dans le texte? Lesquelles ? Cherchez des indices de ces moments-là dans le texte. Ces moments ont-ils quelque chose de particulier pour que vous ayez ressenti des émotions?

\section{Vos émotions suscitées par l'histoire}

Vous avez ressenti des émotions par rapport à l'histoire. Lesquelles ? Ces émotions se sontelles intensifiées (= augmentées) ? A quels moments ? Ont-elles évolué ou se sont-elles transformées? Pour quelles raisons? Quelles émotions avez-vous ressenties à la fin de l'histoire?

\section{Les émotions et votre vitesse de lecture}

$\mathrm{Au}$ cours de votre lecture, avez-vous « ralenti» ou «accéléré » votre vitesse? A quels moments ? Pourquoi ? Est-ce parce que vous avez plus aimé certains passages que d'autres? Est-ce parce que ces derniers ont été plus excitants ou qu'ils ont été plus ennuyants? 


\section{Vos émotions liées à l'écriture du texte}

Avez-vous apprécié ou non, le style de l'auteur (texte raconté à la première personne ou à la troisième, ponctuation, syntaxe et vocabulaire utilisé...)? Expliquez ce que vous avez apprécié ou détesté. Pourquoi ? Relevez les éléments qui vous ont particulièrement plu ou déplu.

\section{Les émotions et votre regard sur le monde}

Pourquoi avoir choisi ce texte? Cette narration vous a-t-elle rappelé une expérience personnelle ? Si oui, laquelle ? Pour des raisons d'ordre culturel ou personnel, vous êtes-vous senti plutôt proche ou carrément étranger à la situation évoquée dans le texte ?

\section{L'évaluation de vos émotions}

Après avoir fini la lecture du texte, parmi les émotions suivantes (plaisir, satisfaction, fierté, tristesse, frustration, colère...) lesquelles ressentez-vous ? Êtes-vous satisfait de la façon dont vous avez ressenti vos émotions par rapport au texte ? Avez-vous éprouvé des difficultés à ressentir certaines émotions liées à l'histoire, aux personnages etc. ? Avez-vous eu des difficultés à ressentir des émotions stylistiques, syntaxiques (la façon dont les phrases sont construites) ou sémantiques (par rapport au vocabulaire) par rapport au texte ? Avez-vous eu une perte de vos émotions? A quels moments ? Avez-vous compris les raisons de cette perte? Était-ce lié à vous-même ou au texte lui-même ? Ressentez-vous des émotions positives ou négatives par rapport à la compréhension de texte en général ? Lesquelles ? Pourquoi?

Le partage des émotions sur le texte, avec vos pairs, a-t-il été bénéfique pour vous ?

Par cette étude, quoiqu'encore insuffisamment développée, nous avons voulu montrer que les émotions faisaient partie intégrante de l'activité de compréhension de textes : «les éléments émotionnels et rationnels sont tissés dans la même trame discursive et s'avèrent, de ce fait, inséparables » (Amossy 172). Nous retiendrons que ce qui prévaut, c'est l'émotion dégagée par la narration littéraire mais la manière dont elle est composée (et qui constitue un des enjeux et l'une des finalités de notre recherche) n'est pas du tout secondaire, puisqu'elle prend sa source dans les émotions mêmes du lecteur, suscitées elles-mêmes par les émotions que les éléments du texte suscitent et donc par l'auteur même, qui, « en jouant avec la structure du texte va jouer avec les émotions du lecteur » (Blanc 156).

Par le biais des textes et au-delà des textes, l'objectif visé par une approche émotionnelle des narrations littéraires est l'acquisition d'une «compétence émotionnelle» dont les huit composantes ont été définies par Saarni (1999) et qui sont les suivantes :

la conscience de ses propres états émotifs qui se réfèrent à la reconnaissance des émotions que l'on ressent, mais aussi aux liens entre ces émotions et les évènements qui les causent; l'habileté à reconnaitre et à comprendre les émotions des autres ; l'habileté à utiliser le vocabulaire associé aux émotions ; la capacité d'empathie, l'empathie étant comprise ici, et comme Legendre (1993) le spécifie, comme la capacité d'identifier les émotions et les représentations d'autrui ; l'habileté à comprendre que l'état émotif interne ne correspond pas nécessairement à ce qui est exprimé ; la capacité à gérer des 
émotions d'aversion ou de détresse en utilisant des stratégies d'autorégulation; la conscience de la nature des relations ou de la communication qui dépend des émotions, les émotions n'étant pas communiquées de la même façon par tout le monde et par toutes les cultures ; et finalement, la capacité d'accepter ses expériences émotives et de développer un sentiment d'autoefficacité. (Lafortune 44)

Pourquoi ne pas nous recentrer sur une classe de langue où l'on saurait raisonner mais où l'on pourrait également ressentir? Un lieu de va-et-vient dialectique entre le « ressentir » et « se regarder ressentir ».

\section{Bibliographie et sitographie}

Abdallah-Pretceille, Martine. « La littérature comme espace d'apprentissage de l'altérité et du divers ». Synergies Brésil n ${ }^{\circ}$ spécial 2 (2010) : 145-155.

Adam, Jean-Michel, dir. Les textes : types et prototypes. Paris : Armand Colin, 2011.

Amossy, Ruth, dir. L'argumentation dans le discours. Paris : Armand Colin, 2010.

Anscombre, Jean-Claude. «Temps, aspect et agentivité dans le domaine des adjectifs psychologiques ». Lidil 32 (2005). http://lidil.revues.org/index110.html [Consulté en juin 2011].

Bandura, Albert. Auto-efficacité. Le sentiment d'efficacité personnelle. Bruxelles : De Boeck, 2003.

Blanc, Nathalie, dir. «Émotion et compréhension de textes ». Émotion et cognition. Quand l'émotion parle à la cognition. Paris : Press, coll. concept-psy, 2006. 123-186.

Bonis (de), Monique. Connaitre les émotions humaines. Hayen : Mardaga, 1996.

Damasio, Antonio, dir. L'erreur de Descartes. La raison des émotions. Paris : Jacob, 2006.

Dufays, Jean-Louis. « La lecture littéraire, des pratiques de terrain aux modèles théoriques ». Lidil 33 (2006). http://lidil.revues.org./index60.html [Consulté en juin 2011].

---, Louis Gemenne et Dominique Ledur, dir. Pour une lecture littéraire. Bruxelles : De Boeck, 2005.

Eco, Umberto, dir. Lector in fabula ou la coopération interprétative dans les textes narratifs. Paris : Grasset, 1985.

Espitalier, Magalie, Anna Tcherkassof et Florian Delmas. «Partage social des émotions et cohésion de groupe ». Les émotions. Cognition, langage et développement. Hayen: Mardaga, 2003. 91-94. 
Gervais, Flore. «Didactique du plaisir de lire : didactique de la littérature jeunesse ». Québec français 100 (1996) : 48-50. http://id.erudit.org/iderudit/58693ac [Consulté en juillet 2011].

Giasson, Jocelyne, dir. Les textes littéraires à l'école. Bruxelles : De Boeck, 2005.

Golder, Caroline et Daniel Gaonac'h, dir. Lire et comprendre. Psychologie de la lecture. Paris : Hachette-Éducation, 2004.

Lafortune, Louise. "Développement de la compétence émotionnelle ». Pédagogie et psychologie des émotions. Vers la compétence émotionnelle. Québec : PU du Québec, coll. Éducation-Recherche, 2009. 35-59.

Langlade, Gérard. «Le sujet lecteur auteur de la singularité de l'œuvre ». Le sujet lecteur. Lecture subjective et enseignement de la littérature. Rennes : PU de Rennes, 2004. 81-91.

Larivey, Michelle. La puissance des émotions. Comment distinguer les vraies des fausses. np : L’Homme, 2002.

Lebrun, Monique, Victor Guérette et Pierre Achim. «L'expérience esthétique des textes au primaire (partie 1)». Québec français 89 (1993): 40-42. http://id.erudit.org/iderudit/44596ac. [Consulté en juillet 2011].

Picard, Michel. La lecture comme jeu. Paris : Minuit, 1986.

Plantin, Christian. «Structures verbales de l'émotion parlée et de la parole émue ». Les émotions. Cognition, langage et développement. Hayen : Mardaga, 2003. 97-130.

Roch-Veiras, Sophie. «Les émotions suscitées par un texte en langue étrangère chez un compreneur-apprenant ». Synergies Espagne 2 (2009) : 223-234.

Rouxel, Annie. «Qu'entend-on par lecture littéraire ? ». La lecture et la culture littéraires au cycle des approfondissements, Actes du colloque de l'université d'automne ClermontFerrand, Royat, du 28 au 31 octobre 2002. CRDP Académie de Versailles, 2004. 1222

--- et Gérard Langlade. « Avant-propos ». Le sujet lecteur. Lecture subjective et enseignement de la littérature. Rennes : PU de Rennes, 2004. 11-16.

Sock, Rudolph et Béatrice Vaxelaire, B. L'anticipation à l'horizon du présent. Hayen : Mardaga, 2004.

Tauveron, Catherine. «La lecture comme jeu, à l'école aussi ». La lecture et la culture littéraires au cycle des approfondissements. Actes du colloque de l'université d'automne Clermont-Ferrand, Royat, du 28 au 31 octobre 2002. CRDP Académie de Versailles, 2004. 23-40. 
Turmel-John, Paule. « Le texte littéraire en classe de seconde ou étrangère ». Québec français 100 (1996) : 51-54. http://id.erudit.org/iderudit/58694ac [Consulté en juillet 2011]. 